Original Article

\title{
COMPARATIVE STUDY BETWEEN OPEN INGUINAL HERNIOPLASTY VERSUS TRANSPERITONEAL LAPAROSCOPY REPAIR
}

\author{
Ibrahem Mohamed Mostafa ${ }^{1}$, Wesam Mohamed Amr ${ }^{1}$, Mostafa Mohamed Khairy ${ }^{1}$, \\ Ayman Omar Abdulaziz Abu Ghamjah' \\ ${ }^{1}$ General Surgery Department, Faculty of Medicine, Zagazig University, Zagazig, Egypt \\ ${ }^{2}$ General Surgery Department, Faculty of Medicine, Tripoli University, Libya
}

Corresponding Author:

Ayman Omar Abdulaziz Abu Ghamjah

General Surgery Department, Faculty of Medicine, Tripoli University, Libya

Aymanaboghmja@gmail.com

$\begin{array}{ll}\text { Submit Date } & 2019-02-23 \\ \text { Revise Date } & 2019-03-17 \\ \text { Accept Date } & 2019-03-18\end{array}$

\section{ABSTRACT}

Background: Successful hernia treatment should offer high patient satisfaction, low cost, low recurrence rate, and rapid return to work Objectives: To compare the effectiveness and safety of laparoscopic and conventional open repair in the treatment of inguinal hernia. To determine whether laparoscopic inguinal hernia repair offers a quicker return to duty over open repair.

Subjects \& Methods: All of the patients were male, both in the group A and group B. Majority of the patients operated were having right inguinal hernia in both groups with right hernia making $80 \%$ in group A and $60 \%$ in group B.

Results: Our study showed that mean hospital stay in days for the group A was significantly lower than the group B ( $1 \pm 0.5$ versus $1.5 \pm 0.5)$. Hence the mean post-operative hospital stay was significantly higher in laparoscopic repair than open hernia repair which was extremely significant.

Conclusion: It can be concluded that laparoscopic hernia repair is associated with high postoperative hospital stay than open hernia repair.

Keywords: Inguinal hernia, Laparoscopic repair, Open repair

\section{INTRODUCTION}

$\mathrm{H}$ ernia is the abnormal exit of an organ or fatty tissue, such as the bowel, through the wall of the cavity in which it normally resides. Repair of inguinal hernia is one of the common surgical procedures done worldwide. Irrespective of country, race or socioeconomic status hernia constitutes a major health-care drain. The definitive treatment of all hernias, regardless of their origin or type, is surgical repair with approximately 20 million repairs done worldwide annually ${ }^{[1]}$. The lack of consensus in the literature as to the optimum repair technique or prosthetic mesh to insure a long term durable result is also surprising ${ }^{[2]}$. Inguinal hernia is a common diagnosis made in the active duty population. In fact the average male carries a lifetime risk of $27 \%$, which appears to drop after 45 years of age ${ }^{[3]}$. Men are seven times more likely to develop an inguinal hernia compared to women ${ }^{[4]}$

There are three important landmarks in the history of repair of inguinal hernia .

1- Tissue repair Eduardo Bassini 1888

2- Onlay mesh Irving Lichtenstein 1984 (tension-free) repair

3- Laparoscopic Ger, Shultz, hernia repair Corbitt, etc. $1990^{[5]}$.

The vast majority of military members are in this demographic. Convalescence following inguinal hernia repair may be significant. Potential loss of work hours because of surgical recovery can be critical in forward deployed units where replacement personnel are not 
readily available. Given the high prevalence of clinically significant hernias and the potential for prolonged recovery periods following repair, the possibility of a shorter convalescence period could be beneficial for an operational medical facility ${ }^{[6]}$.

A laparoscopic approach to inguinal hernia repair may be one potential strategy available to shorten this convalescence period. However, according to the National Hospital Discharge Survey from 2003, only $14 \%$ of all groin hernias in the United States were repaired laparoscopically ${ }^{[7]}$.

The potential benefits of laparoscopic inguinal herniorraphy in the operational setting remain unexplored $^{[6]}$.

The concept of hernia repair underwent evolution with the introduction of monofilament knitted polyethylene plastic mesh. PPM remains most popular both in open and laparoscopic surgery ${ }^{[8]}$.

Indications for surgical repair were based on the operating surgeon's physical exam and clinical judgment. In most cases, these patients had symptomatic inguinal hernias preventing optimal completion of assigned duties ${ }^{[6]}$.

The aim of this study is to compare the effectiveness and safety of laparoscopic and conventional open repair in the treatment of inguinal hernia.

\section{PATIENT AND METHODS}

However, the question about the most appropriate technique still confuses the community of surgeons. Several studies have compared the laparoscopic and open techniques for inguinal hernia repair. The advantages of laparoscopic hernia repair over traditional open repair in terms of limited post-operative pain, early resumption of activity and improved cosmetic have been readily apparent and accepted. Despite excellent long-term outcome after TAPP repair, the use of laparoscopy in hernia repair is still limited [9].
Operating times of surgical techniques varies between surgeons and also vary considerably between centers. It reduces with experience and comparison between laparoscopic and open surgery is subject to bias due to pre-existing familiarity with open techniques ${ }^{[10]}$. It is less important to the patient than a successful operation; the time taken to perform the surgery can have cost implications ${ }^{[11]}$. National Institute for clinical excellence stated that the laparoscopic surgery was associated with a statistically significant increase in operation time compared with open methods of hernia repair ${ }^{[12]}$. Meta-analysis of 16 randomized control trials of trans-abdominal preperitoneal repair demonstrated on overall increase of 13.33 minutes compared with open repair. Meta-analysis of eight randomized control trial of totally extra peritoneal (TEP) repair demonstrated an overall increase of 7.89 minutes compared with open repair.

\section{Operative technique:}

Forty patients were subjected to inguinal hernioplasty, in twenty of them open repair was done while in the other twenty Laparoscopic trans-abdominal periperitoneal repair was done.

\section{Preparation:}

The patient was placed in the supine position on the operating table, urinary catheter was applied and elastic stockings were used. General anesthesia was introduced in all cases. The patient was draped with the whole abdomen, groin, penis and scrotum scrubbed. The surgeon standed on the opposite side of the hernia being repaired with the assistant starting on the hernia side but joining the surgeon once the ports have been placed. The monitor was at the foot on the hernia side, alongside the scrub nurse.

\section{STEP 1: Placement of trocars:}

Pneumoperitoneum was established through a small infra umbilical incision. We 
generally prefer an open technique, in which a blunttipped $12 \mathrm{~mm}$ trocar was inserted into the peritoneal cavity under direct vision. $\mathrm{CO} 2$ was then insufflated into the abdomen to a pressure of 12 to $15 \mathrm{~mm} \mathrm{Hg}$. The angled laparoscope was introduced, and both inguinal areas were inspected. Two $5 \mathrm{~mm}$ ports were placed, one at the lateral border of each rectus abdominis at the level of the umbilicus, to allow placement of the camera and the instruments. The $5 \mathrm{~mm}$ lateral ports may be replaced with $10 \mathrm{~mm}$ ports if only a $10 \mathrm{~mm}$ laparoscope was available.

\section{STEP 2: Identification of anatomic landmarks:}

The four key anatomic landmarks mentioned earlier, the spermatic vessels, the obliterated umbilical artery (medial umbilical ligament), the inferior epigastric vessels (lateral umbilical ligament), and the external iliac vessels; were identified on each side. In the presence of an indirect hernia, the internal inguinal ring was easily identified by the presence of a discrete hole lateral to the junction of the vas deferens, the testicular vessels, and the inferior epigastric vessels. Identification of a direct hernia can be more difficult. Sometimes, a direct hernia appears as a complete circle or hole; at other times, it appears as a cleft, medial to the vas deferens-vascular junction; and at still other times, it was completely hidden by preperitoneal fat and the bladder and umbilical ligaments. Visualization can be particularly difficult in obese patients, who may have considerable lipomatous tissue between the peritoneum and the transversalis fascia, or in patients whose hernia consists of a weakness and bulging of the entire inguinal floor rather than a distinct sac. For adequate definition of this type of hernia and deeper anatomic structures, the peritoneum must be opened, a peritoneal flap developed, and the underlying fatty layer dissected.
Direct hernial defects were often situated medial to the ipsilateral umbilical ligament, and retraction or even division of this structure was sometimes necessary. Division of this structure had no negative sequelae; however, the surgeon should be aware that the obliterated umbilical artery may still be patent and that use of the electrocautery or clips may be necessary. Traction on the ipsilateral testicle can demonstrate the vas deferens when visualization was obscured by overlying fat or pressure from the pneumoperitoneum.

\section{STEP 3: Creation of peritoneal flap:}

Creation of peritoneal flap: The curved scissors or the hook cautery was used to create a peritoneal flap by making a transverse incision along the peritoneum, beginning $2 \mathrm{~cm}$ above the upper border of the internal inguinal ring and extending medially above the pubic tubercle and laterally $5 \mathrm{~cm}$ beyond the internal inguinal ring. Extreme care must be taken to avoid the inferior epigastric vessels. Bleeding from these vessels can usually be controlled by cauterization, but application of haemostatic clips may be necessary on occasion.

Another solution was to pass percutaneously placed sutures above and below the bleeding point while applying pressure to the bleeding vessel so as not to obscure the field of vision. If the monopolar cautery was used to create the peritoneal flap, the entire uninsulated portion of the instrument must be visible at all times to ensure that inadvertent bowel injury did not occur.

The incised peritoneum was grasped along with the attached preperitoneal fat and the peritoneal sac and was dissected cephalad with blunt and sharp instruments to create a lower peritoneal flap. Dissection must stay close to the abdominal wall. A significant amount of preperitoneal fat may be encountered, and this should remain with the peritoneal flap so that the abdominal wall was cleared. When the correct preperitoneal 
plane was entered,dissection was almost bloodless and was easily carried out.

\section{STEP 4: Dissection of hernial sac:}

The hernial sac, if present, was removed from Hesselbach's triangle or the spermatic cord and surrounding muscle through inward traction, counter traction, and blunt dissection with progressive inversion of the sac until the musculofascial boundary of the internal inguinal ring and the key deep anatomic structures were identified. In most cases, the hernial sac can be slowly drawn away from the transversalis fascia or the spermatic cord. The sac was grasped at its apex and pulled inward, thus being reduced by inversion. The indirect sac may be visualized more easily if it was grasped and retracted medially; this step facilitatesits dissection away from the cord structures.

Spermatic cord lipomas usually lay posterolaterally and are extensions of preperitoneal fat. In the presence of an indirect defect, such lipomas should be dissected off the cord along with the peritoneal flap to lie cephalad to the internal inguinal ring and the subsequent repair so that prolapse through the ring can be prevented. A large indirect hernial sac can be divided at the internal ring if it cannot be readily dissected away from the cord structures. This step may prevent the type of cord injury that can result from extensive dissection of a large indirect sac. Division of a large indirect sac was best accomplished by opening the sac on the side opposite the spermatic cord, then completing the division from the inside.

\section{STEP 5: Reidentification and exposure of landmarks:}

Once the peritoneal flap had been created, the key anatomic landmarks mentioned earlier must be reidentified and exposed so that neurovascular structures can be protected from injury and the tissues required for reliable mesh fixation can be located. The pubic tubercle was often more easily felt than seen. Cooper's ligament was initially felt and subsequently seen along the pectineal prominence of the superior pubic ramus as dissection continues laterally and fatty tissue was swept off to expose the glistening white structure. Care must be taken to avoid the numerous small veins that often run on the surface of the ligament, as well as to avoid the occasional aberrant obturator artery. The iliopubic tract was initially identified at the inferior margin of the internal inguinal ring, with the spermatic cord above, and was then followed in both a medial and a lateral direction. Minimal dissection was carried out inferior to the iliopubic tract so as not to injure the genital femoral nerve, the femoral nerve, and the lateral cutaneous nerve of the thigh.

\section{STEP 6: Fixation Of The Mesh:}

Placement of mesh: A $10 \times 6 \mathrm{~cm}$ sheet of polypropylene mesh was rolled into a tubular shape and introduced into the abdomen through the $10 / 12 \mathrm{~mm}$ umbilical trocar. Prolene was preferable to Marlex in this application because it was less dense, conforms more easily to the posterior inguinal wall, and has larger pores, which facilitate visualization and subsequent securing with staples or tacks. The inherent elasticity and resiliency of Prolene mesh allowed it to unroll easily while maintaining its form. The mesh was used to cover the direct space (Hesselbach's triangle), the indirect space, and the femoral ring areas (i.e., the entire inguinal floor). No slit was made in the mesh for the cord. An endoscopic multifire spiral tacker was used to secure the mesh and prevent any migration, beginning medially and proceeding laterally.

The upper margin was first tacked to the rectus abdominis and the transversus abdominis fascia and arch, with care taken to stay 1 to $2 \mathrm{~cm}$ above the level of the internal inguinal ring and to avoid the inferior epigastric vessels, up to a point 
several centimetres lateral to the internal inguinal ring or the indirect hernial defect. Extending mesh fixation to the anterior iliac spine was neither necessary nor desirable. A two-handed technique was used for tack placement: one hand was on the tacker, and the other was on the abdominal wall, applying external pressure to place the wall against the tacker. The tacker itself was frequently pushed against the tissues and used as a spreader and palpator. However, it must not be forced too deeply into the abdominal wall superolateral to the spermatic cord; doing so might lead to inadvertent entrapment of the sensory nerves. The tacker can be moved from the left to the right port, depending on which position more readily allows placement of the staples perpendicular to the mesh and the abdominal wall.

Once the superior margin was fixed, fixation of the inferior margin was accomplished, beginning at the pubic tubercle and moving laterally along Cooper's ligament. The mesh was lifted frequently to ensure adequate visualization of the spermatic cord. Care was taken to avoid the adjacent external iliac vessels, which lied inferiorly. Lateral to the cord structures, all tacks were placed superior to the iliopubic tract to prevent subsequent neuralgias involving the lateral cutaneous nerve of the thigh or the branches of the genitofemoral nerve. If the surgeon can palpate the tacker through the abdominal wall with the non dominant hand, the tacker was above the iliopubic tract. The mesh should lie flat at the end of the procedure.

\section{STEP 7: Closure of peritoneum:}

The peritoneal flap, including the redundant inverted hernial sac, was placed over the mesh, and the peritoneum was reapproximated with the tacker. Reduction of the intra-abdominal pressure to $8 \mathrm{~mm} \mathrm{Hg}$, coupled with external abdominal wall pressure, facilitated a tension free reapproximation. Alternatively, the peritoneum may be sutured over the mesh, but in most surgeons' hands, this closure takes longer.

\section{8: Closure of fascia and skin:}

The peritoneal repair was inspected to ensure that there were no major gaps that might result in exposure of the mesh and subsequent formation of adhesions. The trocars were then removed under direct vision, and the pneumoperitoneum was released. The fascia at the $10 / 12 \mathrm{~mm}$ port sites was closed with 2-0 polydioxanone sutures to prevent incisional hernias. The skin was closed with 4-0 absorbable subcuticular sutures.

\section{Informed consent:}

Written informed consent has been obtained from all individuals included in this study

\section{Ethical approval:}

The research related to human use has been complied with all the relevant national regulations, institutional policies and in accordance the tentes of the ethical guide lines of Zagazig University, and has been approved by the authors institutional review board (IRB). The work should be carried out in accordance with The Code of Ethics of the World Medical Association (Declaration of Helsinki) for studies involving humans.

\section{RESULTS}

\section{Preoperative parameters:}

1. Sex: all patients in the study were adult male patients.

\section{Age:}

The age of the patients included in Group A (Open Mesh Repair) ranged from 17 years to 70 years, with a mean age of $43.9+5$ years. While the age of the patients in Group B (TAPP) ranged from 17 years to 70 years, with a mean age of $44+5$ years.

(1): Comparison between both groups as regard general data.

\section{Personal Data:}

No outstanding difference in both groups as regards the occupation or special habits; 
however there was an increase in the incidence of inguinal hernia among smokers compared to nonsmokers in our study in both groups, as $55 \%$ in group $\mathbf{A}$ and $60 \%$ in group B were smokers, but this result was statistically not significant.

No statistically significant difference could be detected between both groups as regard general data by using chi-square test $\&$ the ttest for the age.

(2): Comparison between both groups as regard presenting symptoms.

(2): Comparison between both groups as regard side of the hernia.

This table shows no significant difference between both groups as regard side of the hernia by using chi-square test.

(3): Distribution of both groups as regard types of hernia.

This table shows no significant difference between both groups as regard type of the hernia by using chi-square test.

\section{Postoperative complications:}

Infection: 4 out of 20 cases i.e. (20\%) were infected in the open group and none in the laparoscopic group, p-value 0.007 .

(4): Distribution Post-Operative Wound Infection
3. Postoperative Hospital stay: Overall, the mean hospital stay in days for the group A was significantly lower than the group B $(1 \pm 0.5$ versus $1.5 \pm 0.5)$.

(5): Distribution Post-Operative hospital stay

\section{Economic cost:}

It is more expensive to perform laparoscopic than open hernia repair. The primary reason for this relates to the cost of extra equipment used for the laparoscopic repair with secondary costs attributed to perceived increases in operating time for the laparoscopic procedure.

5- Cosmoses: In this study 8 out of 20 patients in open group were satisfied by the procedure and 12 patients are not satisfied. All the 20 patients were satisfied cosmetically in laparoscopic group.

(6): Distribution of cosmoses

\section{Detection of recurrence:}

All the cases were followed up periodically for 24 months by clinical and sonological methods. Out of 20 cases one case of recurrent hernia was reported after 1 year in open group and two cases in laparoscopic group.

(7): Recurrence after 1 year

Table 1 Comparison between both groups as regard presenting symptoms.

\begin{tabular}{|c|c|c|c|c|}
\hline Presenting Sympotm & $\begin{array}{c}\text { Group A } \\
\text { No.of } \\
\text { cases (\%) }\end{array}$ & $\begin{array}{c}\text { Group B } \\
\text { No.of } \\
\text { cases (\%) }\end{array}$ & P & $\begin{array}{c}\text { NS } \\
\text { P } \\
\text { VALUE }\end{array}$ \\
\hline Swelling & $14(70 \%)$ & $14(70 \%)$ & 0.774 \\
\hline Pain & $4(20 \%)$ & $3(15 \%)$ & 0.718 \\
\hline Pain and Swelling & $2(10 \%)$ & $3(15 \%)$ & 0.448 & \\
\hline
\end{tabular}

Table 2 Comparison between both groups as regard side of the hernia.

\begin{tabular}{|c|c|c|c|c|}
\hline SIDE OF HERNIA & $\begin{array}{c}\text { Group A } \\
\text { No.of } \\
\text { cases (\%) }\end{array}$ & $\begin{array}{c}\text { Group B } \\
\text { No.of } \\
\text { cases (\%) }\end{array}$ & P & $\begin{array}{c}\text { NS } \\
\text { P } \\
\text { VALUE }\end{array}$ \\
\cline { 1 - 3 } RIGHT & $16(80 \%)$ & $12(60 \%)$ & 0.691 & \\
\hline LEFT & $4(20 \%)$ & $8(40 \%)$ & \\
\hline
\end{tabular}

This table shows no significant difference between both groups as regard side of the hernia by using chi-square test. 
Table 3 Distribution of both groups as regard types of hernia.

\begin{tabular}{|c|c|c|c|c|}
\hline TYPE OF HERNIA & $\begin{array}{c}\text { Group A } \\
\text { No.of } \\
\text { cases (\%) }\end{array}$ & $\begin{array}{c}\text { Group B } \\
\text { No.of } \\
\text { cases (\%) }\end{array}$ & P & $\begin{array}{c}\text { NS } \\
\text { P } \\
\text { VALUE }\end{array}$ \\
\cline { 1 - 3 } Primary direct & $8(40 \%)$ & $6(30 \%)$ & 0.541 & \\
\hline Primary indirect & $12(60 \%)$ & $14(70 \%)$ & & \\
\hline
\end{tabular}

This table shows no significant difference between both groups as regard type of the hernia by using chi-square test.

Table (4): Recurrence after 1 year

\begin{tabular}{|c|c|c|}
\hline $\begin{array}{c}\text { Recurrence } \\
\text { After 1 year }\end{array}$ & Open Group & Laparoscopic Group \\
\hline Yes & $1(5 \%)$ & $2(10 \%)$ \\
\hline No & $19(95.00 \%)$ & $18(90 \%)$ \\
\hline Total & $20(100 \%)$ & $20(100 \%)$ \\
\hline
\end{tabular}

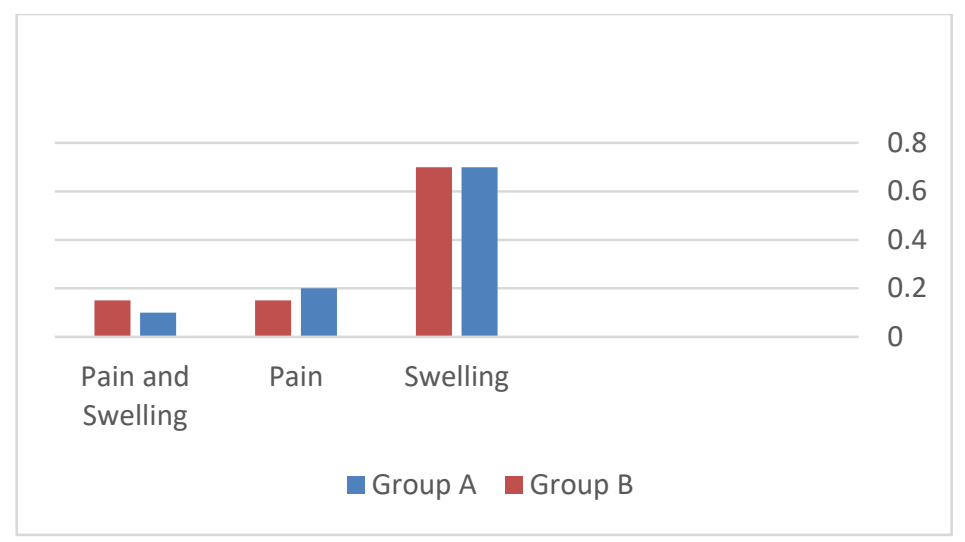

This chart 1 shows that no statistically significant difference could be detected between both groups as regard presenting symptoms by using chi-square test.

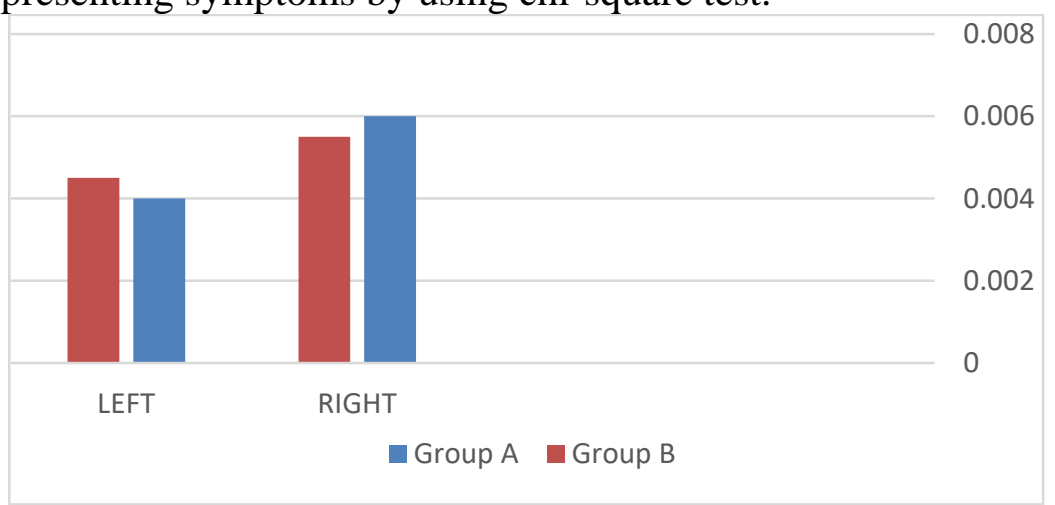

Chart 2 Side of the hernia encountered in the study by percentage. 


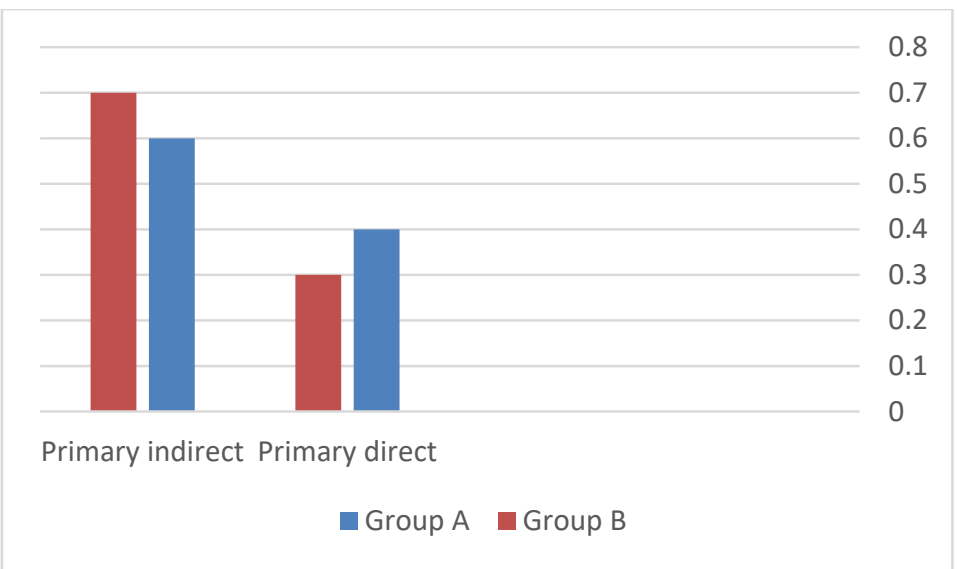

Chart 3 Indicates that majority of cases in each group are primary indirect inguinal hernia.

\section{DISCUSSION}

In this study, all of the patients were male, both in the group A and group B. Majority of the patients operated were having right inguinal hernia in both groups with right hernia making $80 \%$ in group A and $60 \%$ in group B.

As regard operative time there was high significant difference between both techniques with a mean operative time $40 \pm 10$ minutes for group $A$ and $90 \pm 20$ minutes for the group $\mathrm{B}$, thus it is shown that there is around 50 minutes in favor of the group A, which was proved to be statistically significant when proved by the P-value.

Operating times of surgical techniques varies between surgeons and also vary considerably between centers. It reduces with experience and comparison between laparoscopic and open surgery is subject to bias due to pre-existing familiarity with open techniques ${ }^{[10]}$. It is less important to the patient than a successful operation; the time taken to perform the surgery can have cost implications ${ }^{[11]}$. National Institute for clinical excellence stated that the laparoscopic surgery was associated with a statistically significant increase in operation time compared with open methods of hernia repair ${ }^{[12]}$. Meta-analysis of 16 randomized control trials of Trans abdominal pre- peritoneal repair demonstrated on overall increase of 13.33 minutes compared with open repair. Meta-analysis of eight randomized control trial of totally extra peritoneal (TEP) repair demonstrated an overall increase of 7.89 minutes compared with open repair.

Post-operative pain scores were obtained using Visual Analogue Scale (VAS) ${ }^{[13]}$. In this study post-operative pain is significantly more in group A when compared with group B. The pain scores were obtained using visual analogue scale at 24 hours, after 1 week and after 4 week of surgery showed that significantly lower pain score for the group B over the group A on postoperative day 7 , after 4 weeks and 6 month.

A 2003 Cochrane database systematic review demonstrated less persisting pain, and less persisting numbness in the laparoscopic groups. Similarly, another meta-analysis study from the EU Hernia Trialists Collaboration reported decreased post-operative pain with the employment of laparoscopic methods ${ }^{[14]}$. Therefore, there is ample evidence that laparoscopic hernia repair produces less postoperative pain and is associated with similar or less risk of persisting pain than open mesh repair.

In the present study post-operative pain is significantly less in laparoscopic group than open mesh repair group. The difference 
between the two groups was statistically significant. This is in accordance with the last two studies described above. The postoperative pain can further be reduced with the help of newer analgesic techniques like TAPP block, peri-portal infiltration of bupivacaine and advances in fixation devices like glue and self-retaining meshes. The overall incidence of morbidity after laparoscopic groin hernia repair has been quite variable. It is quite possible that complications do occur in any surgical procedure as in the case with laparoscopic hernioplasty, but it is possible to reduce their incidence. Serious complications specific to the laparoscopic technique, although reduced in parallel with training and experience, seen especially in the early stages of hernia surgery and mostly associated with TAPP, have been reported. Complication rates vary from $3 \%$ to $25 \%$ [17].

Incidences of complications after laparoscopic inguinal hernia repair are higher compared with open repair. In MRC hernia trial group, all serious complications occurred in the laparoscopic group ${ }^{\text {16] }}$. In VA trial, complication rate was $39.1 \%$ in lap group including 2 deaths but $33.4 \%$ in open group. In an extensive review by Cochrane group in conjunction with European Hernia trialist group, found serious vascular and visceral injuries more often in laparoscopic group Shoulder pain was transient which got subsided on its own in 1-2 days. The complications regarding wound infection are 4 out of 20 cases i.e. $(20 \%)$ were infected in the open group and none in the laparoscopic group. Study regarding mesh infection might require more number of cases or large case series to analyse and arrive at a definite conclusion.

In the present study, the mean hospital stay in days for the group A was significantly

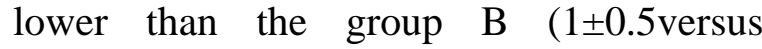
$1.5 \pm 0.5)$. Hence the mean post-operative hospital stay was significantly higher in laparoscopic repair than open hernia repair which was extremely significant. So, from this study it can be concluded that laparoscopic hernia repair is associated with high postoperative hospital stay than open hernia repair.

Studies state that patients have a shorter convalescence and a faster return to work and activities after laparoscopic repair compared to open repair. Data regarding time to return to activity are rather subjective. Type of employment or profession, to which patient is returning will influence how long he needs to be away from work. Patient who is doing desk job in office will return to work earlier than a patent with a job that entails heavy lifting. Time to return to daily activities was found to be shorter for laparoscopic group than those undergoing open repair of hernia in a VA hernia trial group. However, at one month of follow up, there was no difference in the activity level between the laparoscopic and open group. In the present study patients who underwent laparoscopic hernia repair were able to return to their normal work earlier (from 1 to 2 weeks) than those patients who underwent open repair returned to their normal work after 1 month with $\mathrm{p}<0.001$ which is extremely significant. This is a great advantage for Egyptian patients particularly who attend government hospital like ours who earn livelihood on a day to day basis. Most studies mentioned early return to normal work as an advantage of laparoscopic hernia repair, which has been repeated in this study. However, as mentioned, there might not be any difference between the two groups in the level of activity on long-term follow up.

One of the major criticisms of laparoscopic hernia repair is that it is more expensive to perform than open hernia repair. The primary reason for this relates to the cost of 
extra equipment used for the laparoscopic repair with secondary costs attributed to perceived increases in operating time for the laparoscopic procedure ${ }^{[16]}$.

\section{SUMMARY AND CONCLUSION}

Inguinal hernia is a common problem, which can be treated only by surgery. The results support the view that laparoscopic preperitoneal mesh repair is safe and efficient when compared to open mesh repair of inguinal hernia. There is definitive learning curve for surgeons who are newly exposed. The complication rate reduces as the surgeons become more experienced in this procedure comparable with that of open repair. Laparoscopic preperitoneal mesh repair is safe with less post-operative morbidity associated with faster recovery and satisfaction as documented by less postoperative pain, earlier mobilization and discharge from the hospital, as well as early return to work. The hard working below poverty people should be given best treatment, which should allow them to go for their regular work at the earliest and with complete integrity.

As it is costly in private hospitals, the faculty of government hospitals should cater the best treatment at free of cost for poor. To achieve this goal the surgeons should improvise their technical skills in laparoscopic repair of inguinal hernia (TAPP and TEP) with short learning curve. The present study supports the view that laparoscopic pre-peritoneal mesh repair of inguinal hernia is safe and efficacious and offers definitive advantages over open mesh repair and should be an available option for all patients requiring elective hernioplasty.

Declaration of interest

The authors report no conflicts of interest. The authors alone are responsible for the content and writing of the paper.

Funding information

None declared

\section{REFERENCES}

1-Schmidt J, Carbajo MA, Lampert $R$ and Zirngibl H, 2001: laparoscopic intraperitoneal onlay polytetrafluoroethylene mesh repair (IPOM) for inguinal hernia during spinal anesthesia in patients with severe medical conditions. Surg Laparosc Endosc Percutan Tech; 11 (1): 34-37.

2-Trotter D, Lo CH and Grossberg P, 2005: Unusual complications of laparoscopic totally extraperitoneal inguinal hernia repair. ANZ J Surg; 75(10):917-9.

3- Yang C, Zhang H, Pu J, Mei H, Zheng L, Tong Q (2011): Laparoscopic vs open herniorrhaphy in the management of pediatric inguinal hernia: a systemic review and metaanalysis. J Pediatr Surg. 2011;46(9):1824-34.

4- Feliu X, Claveria R, Besora P, Camps J, Sallent FE, Viñas $X$, et al (2011): Bilateral inguinal hernia repair: laparoscopic or open approach? Hernia. 2011;15(1):15-8.

5- Tolba M, Khairi $A$ and Nour-Eldin $O$ (2012): Tension-free mesh inguinal hernia repair, laparoscopic or open? J Mini Inv Surg Sci. 2012;1:149-53.

6- Ansari M and Srivastava V (2014): Comparison of outcomes (early and late) following open and laparoscopic repair of inguinal hernias: an experience of a single surgical unit. Inter J Res Appl Nat Soci Sci. 2014;2(2):163-8.

7- Neumayer L, Hurder GA, Jonasson O, Fitzgibbons R, Dunlop D, Gibbs J, et al. (2004): Open mesh versus laparoscopic mesh repair of inguinal hernia. N Engl J Med. 2004;350(18):1819-27.

8-Wall ML, Cherian T and Lotz JC (2008): Laparoscopic hernia repair the best option? Acta Chir Belg. 2008;108(2):186-91.

9- Smink DS, Paquette IM, Finlayson SR, 2009. Utilization of laparoscopic and open inguinal hernia repair: a population-based analysis. J Laparoendosc Adv Surg Tech. 1;19(6):745-8.

10- Snehal, F, 2008. Laparoscopic versus Open repair of inguinal hernia. World J Laparosc Surg.;1(1):41-8.

11- Felix E, Harbertson N, Vartanian S, 1999. Laparoscopic hernioplasty. Surg Endosc.;13:328-31. 
12- Kumar S, 2002. Chronic pain after laparoscopic and open mesh repair of groin hernia. Br J Surg.;89(11):1476-9.

13- DeLoach LJ, Higgins MS, Caplan AB, Stiff JL, 1998. The VAS in the immediate post-operative period: Intrasubject variability and correlation with a numeric scale. Anesth Analg.;86:102-6.

14- McCormack K, Scott NW, Go PM, Ross S, Grant AM, 2003. EU Hernia Trialists Collaboration. Laparoscopic techniques versus open techniques for inguinal hernia repair. Cochrane Database Syst Rev.;(1):CD001785.

16- McIntosh E, 2001. Cost-utility analysis of open versus laparoscopic groin hernia repair: results from a multicentre randomized clinical trial. Br J Surg. 1;88(5):653-61.

17- Memon MA, Cooper NJ, Memon B, Memon MI, Abrams KR, 2003. Metaanalysis of randomized clinical trials comparing open and laparoscopic inguinal hernia repair. Br J Surg.;90(12):1479-92.

To cite this article: Mostafa IM, Amr WM, KhairyMM, Abu Ghamjah AO. Comparative Study Between Open Inguinal Hernioplasty Versus Transperitoneal Laparoscopy Repair .,Egypt.ZUMJ 2019;25(3);419429,DOI: 10.21608/zumj.2019.9772.1061 